\title{
Bilateral versus unilateral papillary thyroid microcarcinoma: predictive factors and associated histopathological findings following total thyroidectomy
}

\author{
Theodore Karatzas, ${ }^{1}$ Ioannis Vasileiadis, ${ }^{2}$ George Charitoudis, ${ }^{2}$ Efthimios Karakostas, ${ }^{2}$ \\ Sofia Tseleni-Balafouta, ${ }^{3}$ Gregory Kouraklis ${ }^{1}$
}

${ }^{1}$ Second Department of Propedeutic Surgery, School of Medicine, University of Athens, Laikon General Hospital, Athens, ${ }^{2}$ Department of Otolaryngology/Head and Neck Surgery, Venizeleio-Pananeio General Hospital, Herakleion, ${ }^{3}$ Department of Pathology, National and Kapodistrian University of Athens, Athens, Greece

\begin{abstract}
OBJECTIVE: The extent of thyroidectomy for papillary thyroid microcarcinoma (PTMC) is debatable. This study investigated the rate and predictive factors of bilateral versus unilateral PTMC with the objective of identifying those patients who may benefit from total thyroidectomy. DESIGN: Between January 2001 and December 2008, 2019 patients who underwent total thyroidectomy were examined. A total of 319 patients diagnosed histopathologically as PTMC were included in the study. The predictive value of age at diagnosis, gender, tumor size, multifocality, lymph node metastasis, thyroid capsule invasion and nonincidental diagnosis using univariate and multivariate analyses were retrospectively analyzed. RESULTS: Of the 319 patients with PTMC, 77 (24.1\%) presented bilateral disease. In univariate analysis, size of tumor $\geq 5 \mathrm{~mm}(\mathrm{p}<0.001)$, multifocality $(\mathrm{p}<0.001)$, lymph node metastases $(\mathrm{p}<0.001)$, thyroid capsule invasion $(p<0.001)$ and nonincidental diagnosis $(p=0.002)$ were significantly associated with bilaterality. In multivariate analysis, tumor size $(p<0.001)$, multifocality of the primary tumor in the unilateral lobe $(p<0.001)$ and lymph node metastasis $(p<0.001)$ were independent predictive factors for bilateral PTMC. CONCLUSIONS: Tumor size $\geq \mathbf{5 m m}$ and multifocality of the primary carcinoma in the unilateral lobe were independent risk factors for bilateral PTMC. Total thyroidectomy should be considered for these patients, which is of importance for the prediction of possible recurrence of disease.
\end{abstract}

Key words: Bilateral cancer, Papillary thyroid microcarcinoma, Predictive factors, Total thyroidectomy

Address for correspondence:

Theodore Karatzas, Associate Professor of Surgery, Second Department of Propedeutic Surgery, School of Medicine, University of Athens, Laikon General Hospital, Athens, Greece, Tel.: +30 2107462553, E-mail: tkaratz@med.uoa.gr Received 17-01-2013, Accepted 24-03-2013

\section{INTRODUCTION}

Increased awareness of thyroid nodular disease, advances in diagnostic tools and refinements in histopathological criteria have resulted in a marked increase in the number of patients diagnosed with 
thyroid cancer in developed countries. In 2011 there were 48,020 new cases of thyroid cancer and 2,620 deaths due to thyroid cancer in the United States, while American Cancer Society estimates for 2013 are 60,220 new cases and 1850 deaths. ${ }^{1}$ Papillary thyroid carcinoma (PTC) is the most common thyroid follicular cell-derived malignancy and is considered to be a relatively indolent tumor from which death is rare. However, in the elderly and patients exposed to radiation PTC can behave aggressively. ${ }^{2}$

Papillary thyroid microcarcinoma (PTMC) is defined by the World Health Organization (WHO) as a PTC with a maximum diameter of $\leq 10 \mathrm{~mm} .{ }^{3}$ It is estimated that it accounts for up to $30 \%$ of all papillary thyroid cancers, although marked geographic differences in incidence rates have been noted. ${ }^{4,5}$

Most of PTMC are not detectable on clinical examination and they are only diagnosed incidentally during pathologic examination of the thyroid after surgery. The increased accuracy of pathologic examination, in particular thanks to the greater number and thinness of the anatomical slices, has led to more frequent pathologic diagnosis of incidental PTMC. ${ }^{6}$

Additionally, over the last few decades, the widespread use and the technical improvement of thyroid ultrasonography and fine-needle aspiration biopsy (FNAB) have resulted in a marked increase in the rate of preoperative diagnosis of PTMC. ${ }^{7,8}$

However, the management of PTMC, which are often undetectable by palpation, has become a controversial topic. The treatment may range from follow-up without surgery to total thyroidectomy with or without iodine treatment. ${ }^{9-12}$

Although total thyroidectomy is the treatment of choice followed by the majority of surgeons, unilateral lobectomy with radioactive remnant ablation (RRA) is favored for the treatment of PTMC, particularly by American authors. ${ }^{13,14}$ In those patients with PTMC treated by lobectomy, most clinicians would argue for completion thyroidectomy if the risk of PTMC in the contralateral lobe was significant.

In this study, we retrospectively examined the incidence and related risk factors of PTMC with bilateral involvement in an endeavor to determine the independent predictors and to identify those PTMC patients who may benefit from total thyroidectomy.

\section{PATIENTS AND METHODS}

A total of 2019 patients underwent total thyroidectomy during the period between January 2001 and December 2008 in the Department of Otolaryngology in Venizeleio General Hospital of Heraklion, Greece. The medical records of all patients with a final pathologic report of PTMC were reviewed retrospectively.

All patients included in the study underwent standard total thyroidectomy. Surgical management of the patients was recommended upon referral to our hospital for heterogeneous reasons, such as suspicious thyroid malignancy, goiter, hyperthyroidism or compression of neighboring structures. Seventyseven patients underwent total thyroidectomy for suspicious thyroid malignancy (clinically suspected PTMC), 218 for goiter, 16 for hyperthyroidism and 8 for compression of neighboring structures. All patients were operated on by four surgeons, and the pathological evaluation of thyroid specimens was performed by three pathologists at our institution. The entire gland and additional nodal tissue were evaluated from 1-mm-thick anatomical slices.

Of all total thyroidectomy patients, 588 (29.1\%) presented with malignant neoplasms. Papillary carcinoma was diagnosed in $515(25.5 \%)$ patients by histopathological examination, and $319(15.8 \%)$ of these were microcarcinomas.

Inclusion criteria were adult age, standard total thyroidectomy and a histopathological diagnosis of papillary thyroid microcarcinoma according to the World Health Organization criteria. ${ }^{3}$ Exclusion criteria were previous thyroid or parathyroid surgery, previous neck surgery, family history of cancer and history of neck radiation. The patients were divided into two groups: patients in Group 1 had tumor in both thyroid lobes (bilateral PTMC) and patients in Group 2 had tumor localized in only one thyroid lobe (unilateral PTMC).

Our database included age at the time of surgery, gender, surgical procedure, clinical characteristics and final pathological examination of the thyroid carcinoma, including multifocality, bilaterality, cervical lymph node involvement and thyroid capsule invasion. 
The $7^{\text {th }}$ edition of the American Joint Committee on cancer (AJCC) TNM classification of malignant tumors in 2010 was used to describe and categorize cancer stages and progression.

The clinical characteristics considered for statistical analysis were age ( $\leq 45$ years versus $\geq 45$ years), gender (male versus female) and clinically cervical lymph node metastases at the time of diagnosis ( $\mathrm{cN} 0$ versus $\mathrm{cN}+$ ).

The histopathological characteristics considered for statistical analysis were size of the tumor at its greatest diameter $(\leq 5 \mathrm{~mm}$ versus $>5 \mathrm{~mm})$, histopathological evidence of autoimmune thyroid disease (Graves', Hashimoto disease, none), focality of tumor (right lobe versus left lobe, versus bilateral and unifocal versus multifocal).

Multifocal disease was defined as more than one tumor focus in the ipsilateral lobe of the primary tumor. Primary tumor was defined the tumor focus with the largest diameter.

Univariate analysis was performed with the Pearson Chi-square test and Fisher Exact test. The variables that were significantly associated with bilateral PTMC were included in a multivariate logistic regression analysis. Quantitative data are presented as mean \pm SD. A p value $<0.05$ was considered statistically significant. SPSS software (SPSS 17. Inc. Chicago, IL) was used for statistical analysis.

\section{RESULTS}

Of the 515 patients diagnosed with papillary thyroid carcinoma, 319 patients $(62 \%)$ had tumor size measuring $10 \mathrm{~mm}$ or less at maximum diameter and met the inclusion criteria. Of the 319 patients with PTMC, $77(24.1 \%)$ patients had bilateral disease, and $242(75.9 \%)$ had unilateral. A total of $77(22.7 \%)$ patients with PTMC were suspected preoperatively because of a positive FNAB, US findings, suspicious cervical lymph node and/or palpable nodule; these patients constituted the nonincidental group. Fortythree patients were diagnosed by a positive FNAB, 21 patients by US findings and 13 patients by a suspicious cervical lymph node and/or palpable nodule. On univariate analysis, bilaterality was significantly associated with tumor size $(\mathrm{p}<0.001)$, multifocality $(\mathrm{p}<0.001)$, lymph node metastasis $(\mathrm{p}<0.001)$, capsule invasion $(\mathrm{p}<0.001)$ and nonincidental diagnosis $(\mathrm{p}=0.002)$. There were no significant differences between the presence of contralateral carcinoma and gender, age and autoimmune thyroid disease $(p>0.05)$ (Table 1).

We conducted multivariate logistic regression analysis of clinical and pathological factors. The results demonstrated that tumor size $(\mathrm{p}<0.001$, $\mathrm{OR}=3.18)$, multifocality $(\mathrm{p}<0.001, \mathrm{OR}=6.25)$ and lymph node metastases $(\mathrm{p}<0.001, \mathrm{OR}=3.37)$ showed a significant correlation with bilateral PTMC as independent risk factors (Table 2).

Of the 319 patients with PTMC, 261 (81.8\%) were female and $58(18.2 \%)$ were male and the male/female ratio was $1 / 4.1$ (Table 1 ). The mean patient age at surgery was $50.31 \pm 13.03$ years (range from 18 to 88 years). There were 199 (62.4\%) patients over 45 years of age and $120(37.6 \%)$ patients under 45 years of age. The mean diameter of tumor was $4.02 \pm 2.75$ $\mathrm{mm}$ (range from 1 to $10 \mathrm{~mm}$ ). The size of the thyroid cancer was $5 \mathrm{~mm}$ or less in $249(78.1 \%)$ patients and larger than $5 \mathrm{~mm}$ in $70(21.9 \%)$ patients. There was a significant difference in gender between tumor size groups $(\mathrm{p}=0.010)$. Of patients with tumors $5 \mathrm{~mm}$ or less, $209(83.9 \%)$ were female and $40(16.1 \%)$ were male. Among the patients with tumors greater than 5 $\mathrm{mm}, 49(70 \%)$ were female and $21(30 \%)$ were male, who demonstrated a statistically significant association of the size of tumor with male gender $(\mathrm{p}<0.05)$. In the group of patients with tumor size larger than $5 \mathrm{~mm}$, we found that bilaterality was presented in 33 $(42.9 \%)$ patients, multifocality in $44(62.9 \%)$ patients, lymph node metastasis in $18(25.7 \%)$ patients and capsule invasion in $16(22.9 \%)$ patients. On univariate analysis, size of tumor was also strongly associated with bilaterality, multifocality, lymph node metastasis and capsule invasion $(\mathrm{p}=0.001)$.

In $123(38.6 \%)$ patients the tumor was detected in the right lobe, in $119(37.3 \%)$ patients in the left lobe and in $77(24.1 \%)$ patients foci of tumor were detected in both lobes. The presence of multifocality was observed in 103 (32.3\%) patients. Regarding the multifocality, a statistically significant difference was observed between bilateral and unilateral PTMC. Multifocality was found in $68(88.3 \%)$ patients with bilateral PTMC and in 35 (14.5) patients with uni- 
Table 1. Clinical and pathological characteristics of patients with bilateral versus unilateral PTMC ( $\mathrm{x}^{2}$ Pearson test was used - for normally distributed variables mean $\pm \mathrm{SD}$ is reported, for non-normally distributed variables median (range) is reported)

\begin{tabular}{|c|c|c|c|c|c|}
\hline & $\begin{array}{c}\text { Total } \\
\text { patients } \\
(n=319)\end{array}$ & $\begin{array}{l}\text { Bilateral } \\
\text { PTMC } \\
(n=77)\end{array}$ & $\begin{array}{l}\text { Unilateral } \\
\text { PTMC } \\
(\mathbf{n}=\mathbf{2 4 2})\end{array}$ & $\begin{array}{c}\text { Percent }(\%) \\
\text { Total patients / Bilateral PTMC / } \\
\text { Unilateral PTMC }\end{array}$ & P value \\
\hline \multicolumn{6}{|l|}{ Gender } \\
\hline Female & 261 & 61 & 200 & 81.8 / 79.2 / $82.6 \%$ & \\
\hline Male & 58 & 16 & 42 & $18.2 / 20.8 / 17.4 \%$ & 0.876 \\
\hline \multicolumn{6}{|l|}{ Age at diagnosis (years) } \\
\hline Mean \pm SD & $50.31 \pm 13.03$ & $50.10 \pm 13.52$ & $50.38 \pm 12.90$ & & \\
\hline$<45$ & 120 & 31 & 89 & $37.6 / 40.3 / 36.8 \%$ & \\
\hline$\geq 45$ & 199 & 46 & 153 & $62.4 / 59.7 / 63.2 \%$ & 0.734 \\
\hline \multicolumn{6}{|l|}{ Tumor size (mm) } \\
\hline Median (range) & $3(1-10)$ & $6(1-10)$ & $3(1-10)$ & & \\
\hline$\leq 5 \mathrm{~mm}$ & 249 & 44 & 205 & $78.1 / 57.1 / 84.7 \%$ & \\
\hline$>5 \mathrm{~mm}$ & 70 & 33 & 37 & $21.9 / 42.9 / 15.3 \%$ & $<0.001$ \\
\hline \multicolumn{6}{|l|}{ Multifocality } \\
\hline Yes & 103 & 68 & 35 & $32.3 / 88.3 / 14.5 \%$ & $<0.001$ \\
\hline No & 216 & 9 & 207 & $67.7 / 11.7 / 85.5 \%$ & \\
\hline \multicolumn{6}{|l|}{ Autoimmunity } \\
\hline Yes & 201 & 54 & 147 & $67.0 / 70.1 / 60.7 \%$ & 0.175 \\
\hline No & 118 & 23 & 95 & $33.0 / 29.9 / 39.3 \%$ & \\
\hline \multicolumn{6}{|l|}{ Lymph node metastasis } \\
\hline Yes & 34 & 21 & 13 & $10.7 / 27.3 / 5.4 \%$ & $<0.001$ \\
\hline No & 285 & 56 & 229 & $89.3 / 72.7 / 94.6 \%$ & \\
\hline \multicolumn{6}{|l|}{ Thyroid capsular invasion } \\
\hline Yes & 35 & 17 & 18 & $11 / 22.1 / 7.4 \%$ & $<0.001$ \\
\hline No & 284 & 60 & 224 & 89 / 77.9 / $92.6 \%$ & \\
\hline \multicolumn{6}{|l|}{ Mode of diagnosis } \\
\hline $\begin{array}{l}\text { Nonincidental diagnosis } \\
\text { (Clinically suspected) }\end{array}$ & 77 & 31 & 46 & $24.1 / 40.3 / 19 \%$ & 0.002 \\
\hline Incidental diagnosis & 242 & 46 & 196 & 75.9 / 59.7 / $81 \%$ & \\
\hline
\end{tabular}

lateral PTMC. On univariate analysis, multifocality was significantly associated with autoimmune thyroid disease $(p=0.005)$ and size of tumor $(p<0.001)$, bilaterality $(\mathrm{p}<0.001)$, lymph node metastasis $(\mathrm{p}<0.001)$, capsule invasion $(p=0.007)$. No association of multifocality with the age and gender of patients was observed $(p>0.05)$.

Histopathological evidence of autoimmune thyroid disease was diagnosed in $201(67 \%)$ of the patients. Bilaterality $(p=0.014)$, multifocality $(p=0.005)$, size of tumor $>5 \mathrm{~mm}(\mathrm{p}=0.007)$ and lymph node metastasis $(p=0.002)$ were significantly related with the presence of autoimmune thyroid disease at univariate analysis.
The presence of autoimmune thyroid disease did not correlate with gender and age $(\mathrm{p}>0.05)$.

Central neck compartment lymph node dissection was performed in $41(12.9 \%)$ patients and in 34 $(10.7 \%)$ of these patients histopathological nodal involvement was confirmed. At univariate analysis, risk factors for lymph node metastasis were clinically suspected PTMCs $(\mathrm{p}<0.001)$, the presence of bilaterality $(p<0.001)$, tumor multifocality $(p<0.001)$, autoimmune thyroid disease $(\mathrm{p}=0.001)$, capsule invasion $(\mathrm{p}<0.001)$ and size of tumor $>5 \mathrm{~mm}(\mathrm{p}<0.001)$. Age and gender were not associated with lymph node metastasis by univariate analysis $(\mathrm{p}>0.05)$. Multi- 
Table 2. Independent risk factors for bilateral PTMC (multivariate analysis)

\begin{tabular}{lcc}
\hline Variable & p value & $\begin{array}{c}\text { Odds ratio - 95\% } \\
\text { confidence interval }\end{array}$ \\
\hline Gender (male) & 0.859 & $1.05(0.48-2.28)$ \\
Age at diagnosis (years) & 0.902 & $0.86(0.46-1.62)$ \\
$\quad$ 445 years & & \\
Tumor size (mm) & $<0.001$ & $3.18(1.64-6.17)$ \\
$\quad>5$ mm & & \\
Multifocality & $<0.001$ & $6.25(3.45-11.36)$ \\
Autoimmune thyroid disease & 0.664 & $1.38(0.74-2.60)$ \\
Lymph node metastases & $<0.001$ & $3.37(1.43-7.96)$ \\
Thyroid capsular invasion & 0.553 & $1.40(0.55-3.57)$ \\
Nonincidental diagnosis & 0.828 & $1.11(0.50-2.42)$ \\
\hline
\end{tabular}

variate analysis showed that bilaterality $(\mathrm{p}=0.009)$, tumor multifocality $(\mathrm{p}=0.017)$, size of tumor $>5 \mathrm{~mm}$ $(p=0.014)$, presence of capsule invasion $(p=0.012)$ were independently predictive of lymph node metastasis.

Capsule invasion was detected in $35(11 \%)$ patients. Statistically significant difference was found between the two age groups, where capsule invasion was present more frequently in patients under 45 years of age $(\mathrm{p}=0.022)$. Bilaterality $(\mathrm{p}<0.001)$, multifocality $(p=0.002)$, tumor size $(p<0.001)$ and lymph node metastasis $(\mathrm{p}<0.001)$ were also significantly associated with thyroid capsular invasion at univariate analysis. Gender and autoimmune thyroid disease were not associated with higher risk for capsule invasion (Table 1). Age of patient over 45 years $(p=0.019)$ and both tumor size $>5 \mathrm{~mm}$ and lymph node metastasis $(p<0.001)$ were found to be independent risk factors for capsule invasion by logistic regression analysis.

\section{DISCUSSION}

Recent advances in ultrasonography screening and US-guided FNAB have facilitated the detection and diagnosis of PTC and have resulted in a marked increase in the number of patients diagnosed with PTMC. ${ }^{6,7}$ The rate of incidental PTMC that is undetected preoperatively ranges from $1.3 \%$ to $22 \%$ and varies widely, ranging in large series between 4.6 and $100 \%{ }^{11,13}$ This range of incidental PTMC is likely due to the variation in the use of US and US-guided
FNAB and to the experience with these techniques. It has been demonstrated that the biological behavior between incidentally diagnosed and clinically suspected PTMC tumors may vary significantly. ${ }^{8,9}$

While consensus guidelines recommend total thyroidectomy for PTC $\geq 1 \mathrm{~cm}$, the treatment of PTMC is still controversial. As recommended in the NCCN Clinical Practice Guidelines in Oncology: Thyroid carcinoma (v.2.2012): preoperatively detected bilateral PTMC are one of the indications for patients to be treated by total thyroidectomy. However, the optimal approach of surgical resection in cases with preoperatively undetected bilateral PTMC remains a topic of debate.

Studies had reported that the incidence of bilateral PTC ranged from $13 \%$ to $56 \%$, that could be found in total thyroidectomy or completion thyroidectomy, and incidence of bilateral PTMC was approximately $10-30 \% .{ }^{14-17}$ In our study, $24.1 \%$ of PTMC patients who underwent total thyroidectomy had contralateral PTMC. This rate was consistent with other reports. ${ }^{11,14-16}$

In our study, on univariate analysis, bilaterality was significantly associated with the size of tumor $(\mathrm{p}<0.001)$, multifocality $(\mathrm{p}<0.001)$, lymph node metastasis $(p<0.001)$, capsule invasion $(p<0.001)$ and nonincidental diagnosis $(\mathrm{p}=0.002)$ (Table 1$)$. We also demonstrated that multifocality of the primary tumor in the ipsilateral lobe, the size of primary tumor $>5 \mathrm{~mm}$ and the lymph node metastasis at diagnosis were independent predictors for the presence of an incidental contralateral papillary carcinoma and for higher frequency rates of bilaterality (Table 2).

\section{Size of tumor}

Several authors have reported that the presence of contalateral papillary thyroid carcinoma appeared to be unrelated to the size of the primary tumor. ${ }^{16,18,19} \mathrm{By}$ contrast, our study found that primary tumor $\geq 5 \mathrm{~mm}$ was an independent predictive factor for contralateral PTMC. This finding could be explained by the fact that our study included an large number of PTMC patients who all underwent total thyroidectomy compared with other studies that had a relatively smaller sample of patients and only a fraction of the cases underwent total thyroidectomy. ${ }^{18,20}$ Additionally, Zhou et al. 
reported that tumor size $\geq 7 \mathrm{~mm}$ based on US was an independent predictive factor for bilateral PTMC. ${ }^{21}$

\section{Multifocality}

Previous studies have reported comparable findings to our study, suggesting that ipsilateral multifocal disease was predictive of papillary thyroid carcinoma in the contralateral lobe. ${ }^{18-22}$ The multifocal location of the tumor in PTMC is reported to be a risk factor for lymph node metastasis, distant metastases and local recurrence. ${ }^{9,13,15}$ Multifocality is usually found in approximately $30-40 \%$ of PTMC cases ${ }^{14,15,23}$ Both multifocality and lymph node metastasis at diagnosis increased the risk of lateral nodal recurrence, with $11 \%$ of multifocal tumors exhibiting recurrence, compared to $4 \%$ of unifocal tumors. ${ }^{14}$ Additionally, the presence of PTMC foci in both thyroid lobes constituted a risk factor that increased the rate of locoregional recurrence..$^{14,19,24}$

\section{Lymph node metastasis}

The presence of lymph node metastasis in patients with PTMC is frequent. It ranges from 5.2\% to $44.2 \%$ in those patients subjected to therapeutic or necessary dissection, and as high as $29 \%$ to $64 \%$ in patients subjected to prophylactic or elective dissection. ${ }^{25}$ Our study demonstrated that lymph node metastasis at diagnosis was an independent predictor of papillary thyroid carcinoma in the contralateral lobe. Our results confirmed the finding from other studies indicating that multifocality and bilaterality are predictive factors affecting lymph node metastasis. ${ }^{9,15,25}$

In our study, the incidence of lymph node metastasis was $10.7 \%$ overall and decreased to $5.4 \%$ in patients with unilateral PTMC. In contrast, in patients who presented foci in both thyroid lobes, the incidence of lymph node metastasis was elevated to $27.3 \%$. A possible explanation for this result is that bilateral PTMCs were more frequently diagnosed preoperatively after a positive cytology with US-FNAB, or following diagnosis of a cervical nodal metastasis in clinically suspected PTMC (Table 2). In addition, the size of tumor in bilateral PTMC was statistically significantly larger than the size in unilateral PTMC (Table 1). Several authors have reported that PTMCs $>5 \mathrm{~mm}$ had more frequently lymph node metastasis compared with PTMCs $\leq 5 \mathrm{~mm} \cdot{ }^{23,26-28}$ Others stated that there was no difference in aggressiveness between the patients with PTMCs $\leq 5 \mathrm{~mm}$ and those with PTMCs $>5 \mathrm{~mm} \cdot{ }^{15,29}$

\section{Therapy}

Considerable debate has centered on the clinical significance of PTMC and if these tumors should be managed as aggressively as other PTC. It is well known that PTMC have an excellent prognosis with mortality rates of $0-1 \%$. Therefore, unilateral lobectomy for PTMC patients has been advocated by some authors to avoid increased risk of permanent recurrent laryngeal nerve injury and permanent hypocalcemia. ${ }^{14}$ Patients undergoing lobectomy are often subjected to a potential second surgery with a higher rate of complications and nearly $50 \%$ of these may require hormone replacement therapy anyway. ${ }^{30,31}$

Previous studies have maintained that some PTMCs may have an aggressive behavior and can cause local and regional recurrences, as well as cervical lymph node metastases. ${ }^{14}$ Hay et al reported that $80 \%$ of recurrences were within cervical lymph nodes, with most of the remainder representing recurrence in the contralateral nonresected lobe or in the soft tissues of the thyroid bed. ${ }^{14}$ Higher recurrence rates were seen in patients with multifocal tumors and in nodepositive patients. Another risk factor for locoregional recurrence in PTMC patients was the extent of initial surgery. ${ }^{14}$ The recommended strategy of Mayo Clinic suggested that surgery should ensure that all primary tumors are resected and that potential tumor-foci in the contralateral lobe and lymph node of the central neck compartment are resected at the same operation if this can achieved without unjustified risk.

The same surgical strategy is adopted by our department. We prefer total thyroidectomy to lobectomy because of the likelihood of bilaterality and also because in the cases where a completion thyroidectomy was required, the risk of complications was much higher. Moreover, the advantages of total thyroidectomy are the improved ablation of the tumor, greater sensitivity of thyroglobulin, allowance for use of I-131 in the detection of metastasis and recurrence and also lower local recurrence rate because all potential foci in both lobes are removed. ${ }^{4,28}$ Of note, total thyroidectomy allows the pathologist to assess the entire thyroid gland and to diagnose bilaterality, 
multifocality and histological variants of tumor and extrathyroidal extension.

Finally, an important reason for considering total thyroidectomy over lobectomy is the reported low frequency of permanent recurrent laryngeal nerve damage or permanent hypocalcemia when performed by experienced surgeons. ${ }^{14,32}$ The complications rate in this series was relatively low, with 11 patients (3.4\%) experiencing transient hypocalcemia, 3 patients $(0.9 \%)$ experiencing permanent hypocalcemia and 4 patients $(1.2 \%)$ presenting transient unilateral recurrent laryngeal nerve palsy. The low rate of complications associated with the complete surgery of bilateral resection, which eliminates the risk of cancer recurrence observed with unilateral lobectomy when tumor bilaterality exists, are the main rationale for our center adopting total thyroidectomy as the treatment of choice for PTMC patients.

The limitations of our study include the retrospective nature of analysis with patients from a single institution, the fact that histology was interpreted by three pathologists, the absence of post-operative scan to assess the completeness of total thyroidectomy, the exclusion of patients with a family history of cancer and previous neck radiation and the lack of patients' follow-up.

\section{CONCLUSION}

The high rate $(24.1 \%)$ of bilateral involvement of PTMC patients in this study determined our strategy to carry out surgical resection. Tumor size $\geq 5 \mathrm{~mm}$ and multifocality of the primary carcinoma in the unilateral lobe were independent risk factors for bilateral PTMC. We suggest that total thyroidectomy should be considered in PTMC patients in whom tumor size $\geq 5 \mathrm{~mm}$ and multifocality are detected preoperatively, since this approach is important for the prediction of possible recurrence of disease.

\section{REFERENCES}

1. Siegel R, Ward E, Brawley O, et al, 2011 Cancer statistics, 2011:the impact of eliminating socioeconomic and racial disparities on premature cancer deaths.CA Cancer J Clin 61: 212-236.

2. Mazzaferri EL, 1987 Papillary thyroid carcinoma: factors influencing prognosis and current therapy. Semin
Oncol 14: 315-332.

3. Livolsi VA, Saavedra JA 2004 Papillary carcinoma. In: De Lellis RA, Lloyd RV, Heitz PU, Eng C, editors. World Health Organization. Classification of tumours. Pathology and genetics of tumours of endocrine organs. Lyon, France: IARC Press; pp, 57-66.

4. Ito Y, Uruno T, Nakano K, et al, 2003 An observational trial without surgical treatment in patients with papillary microcarcinoma of the thyroid. Thyroid 13: 381-387.

5. Haymart DT, Cayo M, Chen H, 2009 Papillary thyroid microcarcinomas: big decision for a small tumor. Ann Surg Oncol 16; 3132-3139.

6. Grodski S, Brown T, Sidhu S, et al, 2008 Increased incidence of thyroid cancer is due to increased pathologic detection. Surgery 144: 1038-1043.

7. Davies L, Welch HG, 2006 Increasing incidence of Thyroid cancer in United States, 1973-2002. JAMA 295: 2164-2167.

8. Lo CY, Chan WF, Lang BH, et al, 2006 Papillary microcarcinoma: is there any difference between clinically overt and occult tumors? World J Surg 30: 759-766.

9. Ito Y, Tomoda C, Uruno T, et al, 2004 Papillary microcarcinoma of the thyroid: how should it be treated? World J Surg 28: 1115-1121.

10. Mazzaferi EL, 2006 Managing small thyroid cancers. JAMA 295: 2179-2182.

11. Miccoli P, Minuto MN, Galleri D, et al, 2006 Incidental thyroid carcinoma in a large series of consecutive patients operated for benign thyroid disease. ANZ J Surg 76: 123-126.

12. Bilimoria KY, Bentrem DJ, Ko CY, et al, 2007 Extent of surgery affects survival for papillary thyroid cancer. Ann Surg 246: 375-381.

13. Roti E, degli Uberti EC, Bondanelli M, et al, 2008 Thyroid papillary micocarcinoma: a descriptive and meta-analysis study. Eur J Endocrinol 159: 659-673.

14. Hay ID, Hutchinson ME, Gonzalez-Losada, T et al, 2008 Papillary thyroid microcarcinoma: a study of 900 cases observed in a 60-year period. Surgery 144: 980-988.

15. Chow SM, Law SC, Chan JK, et al, 2003 Papillary microcarcinoma of the thyroid-prognostic significance of lymph node metastasis and multifocality. Cancer 98: 31-40.

16. Pitt SC, Sippel RS, Chen H, 2009 Contralateral papillary thyroid cancer: does size matter? Am J Surg 197: 342-347.

17. Hwang E, Pakdaman MN, Tamilia M, et al, 2010 Bilateral papillary thyroid cancer and associated histopathological findings. J Otolaryngol Head Neck Surg 39: 284-287.

18. Kim ES, Kim TY, Koh JM, et al, 2004 Completion thyroidectomy in patients with thyroid cancer who initially underwent unilateral operation. Clin Endocrinol 61: 145-148.

19. Mazzaferri EL, Jhiang SM, 1994 Long-term impact of initial surgical and medical therapy on papillary and follicular thyroid cancer. Am J Med 97: 418-428.

20. Koo BS, Lim HS, Lim YC et al, 2010 Occult contralateral 
carcinoma in patients with unilateral papillary thyroid microcarcinoma. Ann Surg Oncol 17: 1101-1105.

21. Zhou YL, Gao EL, Zhang W, et al, 2012 Factors predictive of papillary thyroid micro-carcinoma with bilateral involvement and central lymph node metastasis: a retrospective study. World J Surg Oncol 10: 67.

22. Connor MP, Wells D, Schmallbach CE, 2011 Variables predictive of bilateral occult papillary microcarcinoma following total thyroidectomy. Otolaryngol Head Neck Surg 144: 210-215.

23. Vasileiadis I, Karakostas E, Charitoudis G, et al, 2012 Papillary thyroid microcarcinoma: clinicopathological characteristics and implications for treatment in 276 patients. Eur J Clin Invest 42: 657-664.

24. Baudin E, Travagli JP, Ropers J, et al, 1998 Microcarcinoma of the thyroid gland: the Gustave-Roussy Institute experience. Cancer 83: 553-559.

25. Wada N, Duh QY, Sugino K, et al, 2003 Lymph node metastasis from 259 papillary thyroid carcinomas. Frequency, pattern of occurrence and recurrence and optimal strategy for neck dissection. Ann Surg 237: 399-407.

26. Kim BY, Jung CH, Kim JW, et al, 2012 Impact of clinicopathologic factors on subclinical central lymph node metastasis in papillary thyroid microcarcinoma. Yonsei Med J 53: 924-930.

27. Miccoli P, Minuto MN, Ugolini C, et al, 2007 Intrathyroidal differentiated thyroid carcinoma: tumor size-based surgical concepts. World J Surg 31: 888-894.

28. Friguglietti CU, Dutenhefner SE, Brandao LG, et al, 2011 Classification of papillar thyroid microcarcinoma according to the size and fine-needle aspiration cytology: Behavior and therapeutic implications. Head Neck 33: 696-701.

29. Arora N, Turbedian HK, Kato MA, et al, 2009 Papillary thyroid carcinoma and microcarcinoma: is there a need to distinguish the two? Thyroid 19: 473-477.

30. McDougall IR, Camargo CA, 2007 In my view. Treatment of micropapillary carcinoma of thyroid: where do we draw the line? Thyroid 32: 279-281.

31. Farkas EA, King TA, Bolton JS, et al, 2002 A comparison of total thyroidectomy and lobectomy in the treatment of dominant thyroid nodules. Am Surg 68: 678-682.

32. Sosa JA, Bowman HA, Tielsch JM, et al, 1998 The importance of surgeon experience for clinical and economics outcomes for thyroidectomy. Ann Surg 228: 320-330. 